\title{
Ionospheric response to magnetar flare: signature of SGR J1550-5418 on coherent ionospheric Doppler radar
}

\author{
Ayman Mahrous \\ Space Weather Monitoring Center, Physics Dept., Faculty of Science, Helwan University, Ain Helwan, Cairo 11795, Egypt \\ Correspondence to: Ayman Mahrous (amahrous@cern.ch)
}

Received: 27 July 2016 - Revised: 8 February 2017 - Accepted: 13 February 2017 - Published: 7 March 2017

\begin{abstract}
This paper presents observational evidence of frequent ionospheric perturbations caused by the magnetar flare of the source SGR J1550-5418, which took place on 22 January 2009. These ionospheric perturbations are observed in the relative change of the total electron content $(\Delta \mathrm{TEC} / \Delta t)$ measurements from the coherent ionospheric Doppler radar (CIDR). The CIDR system makes high-precision measurements of the total electron content (TEC) change along raypaths from ground receivers to low Earth-orbiting (LEO) beacon spacecraft. These measurements can be integrated along the orbital track of the beacon satellite to construct the relative spatial, not temporal, TEC profiles that are useful for determining the large-scale plasma distribution. The observed spatial TEC changes reveal many interesting features of the magnetar signatures in the ionosphere. The onset phase of the magnetar flare was during the CIDR's nighttime satellite passage. The nighttime small-scale perturbations detected by CIDR, with $\Delta$ TEC/ $\Delta t \geq 0.05$ TECU s $^{-1}$, over the eastern Mediterranean on 22 January 2009 were synchronized with the onset phase of the magnetar flare and consistent with the emission of hundreds of bursts detected from the source. The maximum daytime large-scale perturbation measured by CIDR over northern Africa and the eastern Mediterranean was detected after $\sim 6 \mathrm{~h}$ from the main phase of the magnetar flare, with $\Delta \mathrm{TEC} / \Delta t \leq 0.10 \mathrm{TECU} \mathrm{s}^{-1}$. These ionospheric perturbations resembled an unusual poleward traveling ionospheric disturbance (TID) caused by the extraterrestrial source. The TID's estimated virtual velocity is $385.8 \mathrm{~m} \mathrm{~s}^{-1}$, with $\Delta \mathrm{TEC} / \Delta t \leq 0.10 \mathrm{TECU} \mathrm{s}^{-1}$.
\end{abstract}

Keywords. Ionosphere (ionospheric disturbances) - radio science (radar astronomy) - solar physics astrophysics and astronomy ( $\mathrm{X}$ rays and gamma rays)

\section{Introduction}

Magnetars are a subclass of isolated neutron stars possessing the most extreme magnetic fields in the universe with $B>10^{14}-10^{15} \mathrm{G}$ (Thompson and Duncan, 1995, 1996). The magnetar model (Thompson et al., 2002) identified the power source of these objects to be the decay of their strong magnetic fields. Originally classified as two distinct types of objects, anomalous X-ray pulsars (AXPs) and soft gamma repeaters (SGRs) are currently believed to be of the magnetar class (for reviews, see Woods and Thompson, 2006; Mereghetti, 2008). In addition to being bright X-ray sources, SGRs and AXPs emit intense bursts in hard X-rays and soft gamma rays at a highly unpredictable frequency with peak luminosities ranging from $10^{38}$ to $>10^{47} \mathrm{erg} \mathrm{s}^{-1}$, which is considered as the observational signature of magnetars (Duncan and Thompson, 1992; Kouveliotou et al., 1998). They are known to exhibit emissions as sporadic bursts, which are classified into three kinds according to their luminosities and durations: giant flares, intermediate flares, and short bursts. The X-ray source 1E 1547.0-5408, also known as SGR 1550-5418, was discovered in 1980 with the Einstein satellite as a point source (Lamb and Markert, 1981). The discovery of radio pulsations with a period of $2.1 \mathrm{~s}$ and a period derivative of $2.3 \times 10^{-11} \mathrm{~s} \mathrm{~s}^{-1}$ confirmed its AXP classification (Camilo et al., 2007). It was identified only recently as a magnetar by Gelfand and Gaensler (2007) based on its $\mathrm{X}$-ray spectrum and infrared flux. The dipole surface magnetic field strength and characteristic age are estimated to be about $3.2 \times 10^{14} \mathrm{G}$ and $0.69 \mathrm{kyr}$, respectively (Dib et al., 2012). These features make this object relatively young and classify it as one of the fastest known rotating magnetars.

In early October 2008, both the Swift Burst Alert Telescope (BAT) and the Fermi Gamma Ray Burst Monitor (GBM) were triggered by numerous bursts from the source 
SGR 1550-5418 (Israel et al., 2010; von Kienlin et al., 2012). The source entered a second, even more active phase on 22 January 2009, during which a large number of bursts were observed by several satellites, as detected by Swift (Gronwall et al., 2009), Fermi GBM (Connaughton and Briggs, 2009; von Kienlin and Connaughton, 2009), Konus-Wind (Golenetskii et al., 2009), and RHESSI (Bellm et al., 2009). BAT was triggered on 22 January 2009 at 01:32:41 UT and the first X-Ray telescope (XRT) observation on the Swift satellite began about 50 min later. The peak of the persistent emission occurred $\sim 6 \mathrm{~h}$ after BAT was first triggered. This was accompanied by a hardening of the spectrum in the $1 \times 10 \mathrm{keV}$ band (see a summary of the observations in Table 1 in Scholz and Kaspi, 2011). The wide-band allsky monitor (WAM) onboard the Suzaku satellite detected at least 254 bursts in the $0.16-6.2 \mathrm{MeV}$ band over the period of 22 January 2009 00:57-17:02 UT from the direction of the source. One of these bursts, which occurred at 06:45:13 UT, produced the brightest fluence in the 0.5$6.2 \mathrm{MeV}$ range, with an averaged $0.16-6.2 \mathrm{MeV}$ flux and extrapolated $25 \mathrm{keV}-2 \mathrm{MeV}$ fluence of about $1 \times 10^{-5}$ and about $3 \times 10^{-4} \mathrm{erg} \mathrm{cm}^{-2}$, respectively (Terada et al., 2009). Mereghetti et al. (2009) reported on the observations obtained by the INTEGRAL satellite on 22 January 2009 with the emission of hundreds of bursts in a time span of a few hours, starting at 02:46 UT. The peak of the bursting rate occurred around 06:48 UT, when more than 50 bursts were recorded in $10 \mathrm{~min}$. The total fluence measured from the 125 bursts emitted from 04:30 to 07:00 UT was $5.2 \times$ $10^{4} \mathrm{erg} \mathrm{cm}^{2}(25 \mathrm{keV}-2 \mathrm{MeV})$. This activity was observed by several high-energy missions, creating a good opportunity for investigating the broadband spectra of magnetar short bursts and intermediate flares in detail. Broadband spectral properties have been reported by several authors (e.g., van der Horst et al., 2012; Lin et al., 2012; Younes et al., 2014).

Earth's ionosphere can be thought of as a gigantic detector that responds to the ionizing radiation emitted through high-energy astrophysical phenomena without interruption such as Earth occultation (Mondal et al., 2012). Soft X-ray emissions from solar flares are the more common sources of ionospheric disturbances, which can be monitored using the very low frequency (VLF) technique (Bracewell and Straker, 1949; Thomson et al., 2005; Pacini and Raulin, 2006; Raulin et al., 2006, 2010). In addition to these solarterrestrial events, the lower ionosphere is also affected by high-energy photons ( $\mathrm{X}$-rays and gamma rays) from extraterrestrial sources like gamma ray burst (GRB) and SGR (Inan et al., 1999, 2007; Tanaka et al., 2010). Cosmic gamma rays play an important role in ionizing the neutral atmosphere through electromagnetic cascading (Mahrous and Inoue, 2002). The ionospheric disturbance caused by a cosmic gamma ray burst was first reported by Fishman and Inan (1988). It suggested that gamma rays deposit their energies in the lower ionosphere, abnormally ionize the neutral atmosphere there, and modify the electron density height profile. So far the detection of ionization excesses by using VLF observations has only been reported for four extraterrestrial events (for summary see Raulin et al., 2014).

Many authors observed and reported the first observation of the source SGR J1550-5418 through VLF perturbation on 22 January 2009. The South America VLF Network (SAVNET; Raulin et al., 2009) clearly showed sudden amplitude and phase changes at the corresponding times of eight of these X-ray bursts (Tanaka et al., 2010). Mondal et al. (2012) found convincing evidence that the lower ionospheric height went down significantly by about $15 \mathrm{~km}$ during that event. They also computed the evolution of the electron number density of the ionosphere due to that event and found that the ionosphere was becoming increasingly charged due to repeated bombardment of the high-energy radiations. Raulin et al. (2014) detected an ionospheric disturbance during the event, which was revealed by the simultaneous phase and amplitude records from two VLF propagation paths between the transmitter NPM (Hawaii) and the receivers ROI (Brazil) and EACF (Antarctic Peninsula). Although the previously mentioned authors have specifically studied the temporal sudden ionospheric disturbance (SID) due to the magnetar flare, there was no information about the spatial ionospheric perturbations, sometimes called the traveling ionospheric disturbance (TID), caused by the event.

The TIDs are understood as plasma density fluctuations that propagate through the ionosphere at an open range of velocities and frequencies. The trends of such fluctuations can be seen in most of the ionosphere measurement techniques (Hernández-Pajares, et al., 2006). However, the exact generation mechanisms of TIDs, such as Joule heating and Lorentz force (e.g., Oyama and Watkins, 2012) are only poorly understood because of several interaction mechanisms with electric and magnetic fields as well as thermospheric winds.

In this paper, we introduced the spatial, not temporal, ionospheric disturbance during the gigantic extraterrestrial event from the source SGR J1550-5418 that took place on 22 January 2009. The unusual TID parameters due to that magnetar flare, such as direction and speed are estimated. The paper is divided into four sections. We describe the instrumentation in Sect. 2, the discussion in Sect. 3, and finally the conclusions in Sect. 4.

\section{Instrumentation}

The Ionospheric Tomography Network of Egypt (ITNE) is a chain of passive UHF-VHF (ultra-high-frequency) receivers, known as coherent ionospheric Doppler radars (CIDRs). The first ITNE CIDR was installed in May 2008 at Helwan (geographic latitude $29.9^{\circ}$, longitude $31.3^{\circ}$ ) (Mahrous et al., 2010). CIDR receivers make high-precision (on the order of $\left.10^{4} \mathrm{TECU} \mathrm{s}^{-1}\right)\left(1 \mathrm{TEC}\right.$ unit $(\mathrm{TECU})=10^{16}$ electrons $\left.^{-2}\right)$ measurements (where TEC is total electron content) between rays underneath low Earth-orbiting (LEO) spacecraft. Each 


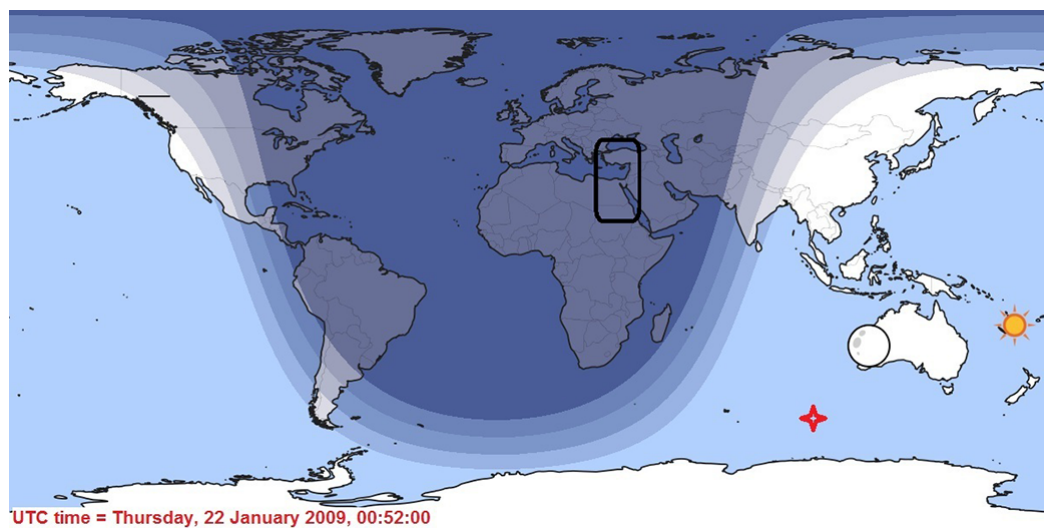

Figure 1. Map shows the solar nighttime and daytime conditions (dark blue and light blue areas, respectively) on 22 January 2009 at 00:52 UT, the projection on the surface of the Earth of the Sun's position (yellow circle), moon's position (white circle), and the sub-flare point (red star). The black rectangle defines the field of view of CIDR, as indicated by the satellite tracks in Fig. 2.

receiver measures the Doppler shift in 150 and $400 \mathrm{MHz}$ signals at a $1 \mathrm{~s}$ data rate. A linear combination of these Doppler shifts reveals relative change of the total electron content $(\Delta \mathrm{TEC} / \Delta t)$. These measurements can be integrated along the orbital track of the beacon satellite, flying between 700 and $1100 \mathrm{~km}$, to construct the relative TEC profile at a $1 \mathrm{~s}$ data rate (relative to an unknown integration constant). The derived TEC values are useful for determining the largescale plasma distribution, but the $\Delta \mathrm{TEC} / \Delta t$ data are more sensitive to small-scale phenomena and wave activity. The CIDR system has many advantages over the Global Navigation Satellite System (GNSS). Because CIDR's LEO spacecraft fly at lower orbits, with an altitude range of 521 to $1158 \mathrm{~km}$, the measured TEC is purely ionospheric, as opposed to GNSS TEC measurements, which have a minor plasmaspheric component. In addition, LEO satellites cross the receiver's field of view in less than $15 \mathrm{~min}$; therefore, the structures with $\Delta \mathrm{TEC} / \Delta t$ are usually treated as spatial not temporal variations. CIDRs and their measurements are discussed in greater detail by Garner et al. $(2008,2009)$.

\section{Discussion}

This study examines five satellite passes observed by CIDR during the SGR J1550-5418 burst on 22 January 2009. We add one additional pass to show the recovery phase of the magnetar flare on 23 January 2009. The space weather data show that 22 January 2009 was a geomagnetically quiet day. The total $\mathrm{Kp}$ index was 2.0 (http://wdc. kugi.kyoto-u.ac.jp/kp/index.html), and the $\mathrm{F} 10.7 \mathrm{~cm}$ solar radio flux was $70.1 \times 10^{22} \mathrm{~W} \mathrm{~m}^{-2} \mathrm{~Hz}$ (http://eng.sepc.ac.cn/ F107Index.php). Figure 1 shows the solar terminator during the SGR burst on 22 January 2009 at 00:52 UT. The dark and light blue areas indicate the regions under night time conditions and under solar illumination, respectively. The white and yellow circles represent the projections of the moon and the Sun, respectively, on the Earth's surface. The point on the Earth directly beneath the flare (sub-flare point) was located at $54.3^{\circ} \mathrm{S}, 14.0^{\circ} \mathrm{E}$ (Tanaka et al., 2010), and its position is defined by a red star. The black rectangle shows the field of view of CIDR, corresponding to the satellite tracks in Fig. 2. The onset phase of the magnetar flare was during the CIDR's nighttime satellite passage. Figure 2 shows a set of maps of the CIDR's passing satellites over northern Africa and the eastern Mediterranean region. The satellite tracks are shown in solid lines and the ionospheric pierce point (i.e., the intersection of the receiver satellite line of sight with the ionosphere, assumed as a thin layer at a fixed height) is shown in thick lines on the map, respectively. The CIDR's passing satellites began at 01:21 UT on 22 January 2009 and ended on 23 January 2009 at 04:10 UT. Half of the satellite passes were during the local nighttime (indicated by black filled circles in the bottom right corner of each map), while the rest were during the daytime (open white circles). The $\mathrm{F}$ region pierce point is set to $350 \mathrm{~km}$ and the obliquity factor is only used for azimuth angles within $30^{\circ}$ of an overhead pass. The geomagnetic equator is offset $7-8^{\circ}$ north of the geographic equator in the Egyptian sector.

Figure 3 shows the vertical total electron content (VTEC) along the satellite tracks shown in Fig. 2 as a function of the geographic latitude. The VTEC latitudinal distribution at the $350 \mathrm{~km}$ intercept resembles a logarithmic profile. The complete profile of the equatorial anomaly is shown in panels a, c, and $d$. The gradual reduction of VTEC from 22 to $30^{\circ}$ geographic latitude (from 15 to $23^{\circ}$ magnetic latitude) suggests that radio rays are passing through the equatorward side of the northern equatorial fountain peak (see Mahrous et al., 2010, for details). The total VTEC gradient was slightly larger than $5 \times 10^{-2}$ TECU per degree, as measured by the OSCAR 25 satellite overpass. A larger TEC gradient is observed in those local sunrise passes (panels $c, d$, and e) than was seen during the previous nighttime passes (panels $a, b$, 


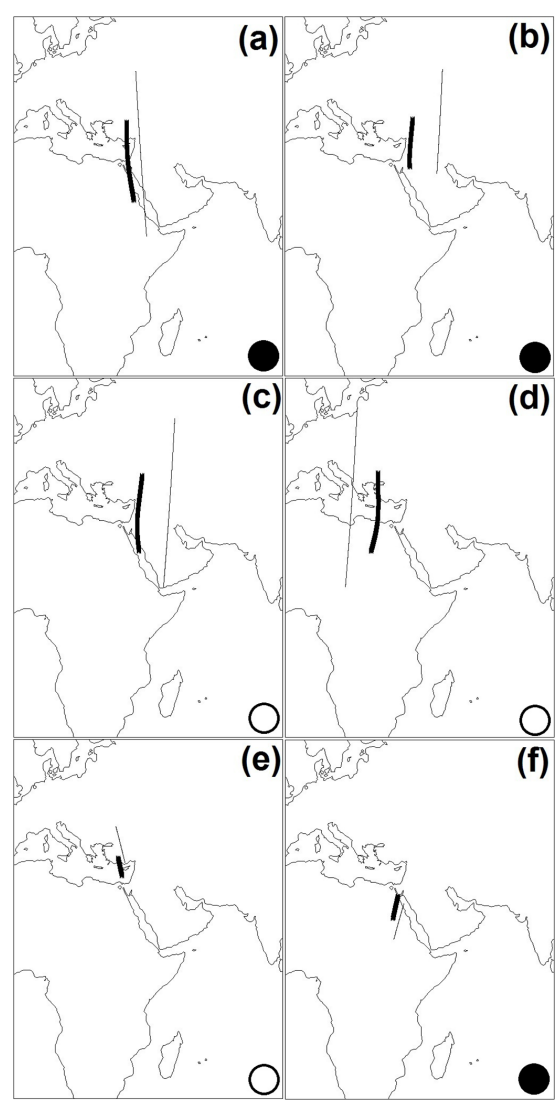

Figure 2. Set of maps show the satellite track (thin line) and the $F$ region (thick line) pierce points over northern Africa and the eastern Mediterranean. The local night and day times during the satellite passes are indicated by black filled and white open circles at the bottom right corner of each map. The CIDR passes (alphabetically sequenced from a to f) were detected on 22 January 2009 at 01:21, 02:56, 12:48, 14:36, 15:44 UT and on 23 January 2009 at 04:10 UT, respectively.

and f). This enhancement is over 3 times larger than the enhancement observed during the morning pass.

Figure 4 shows the relative change in the total electron content $\Delta \mathrm{TEC} / \Delta t$ along the corresponding satellite tracks shown in Fig. 2 as a function of the geographic latitude. The VTEC latitudinal distribution at the $350 \mathrm{~km}$ intercept resembles a logarithmic profile. The first CIDR passage (Fig. 4a) showed that the ionosphere was remarkably smooth on 22 January 2009 at 01:21 UT. Both BAT and INTEGRAL were triggered with the emission of hundreds of bursts in the band (25 keV-2 MeV) from the source SGR 1550-5418 at 01:32:41 and 02:46 UT, respectively (see Scholz and Kaspi, 2011; Mereghetti et al., 2009). Nearly $10 \mathrm{~s}$ after INTEGRAL was triggered, the CIDR started to detect small-scale ionospheric disturbances over the eastern Mediterranean between 30.5 and $41^{\circ}$ geographic latitude at 02:56 UT (Fig. 4b), with $\Delta \mathrm{TEC} / \Delta t \geq 0.05 \mathrm{TECU} \mathrm{s}^{-1}$. The peaks of the bursting rate detected by BAT, Suzaku, and INTEGRAL occurred around
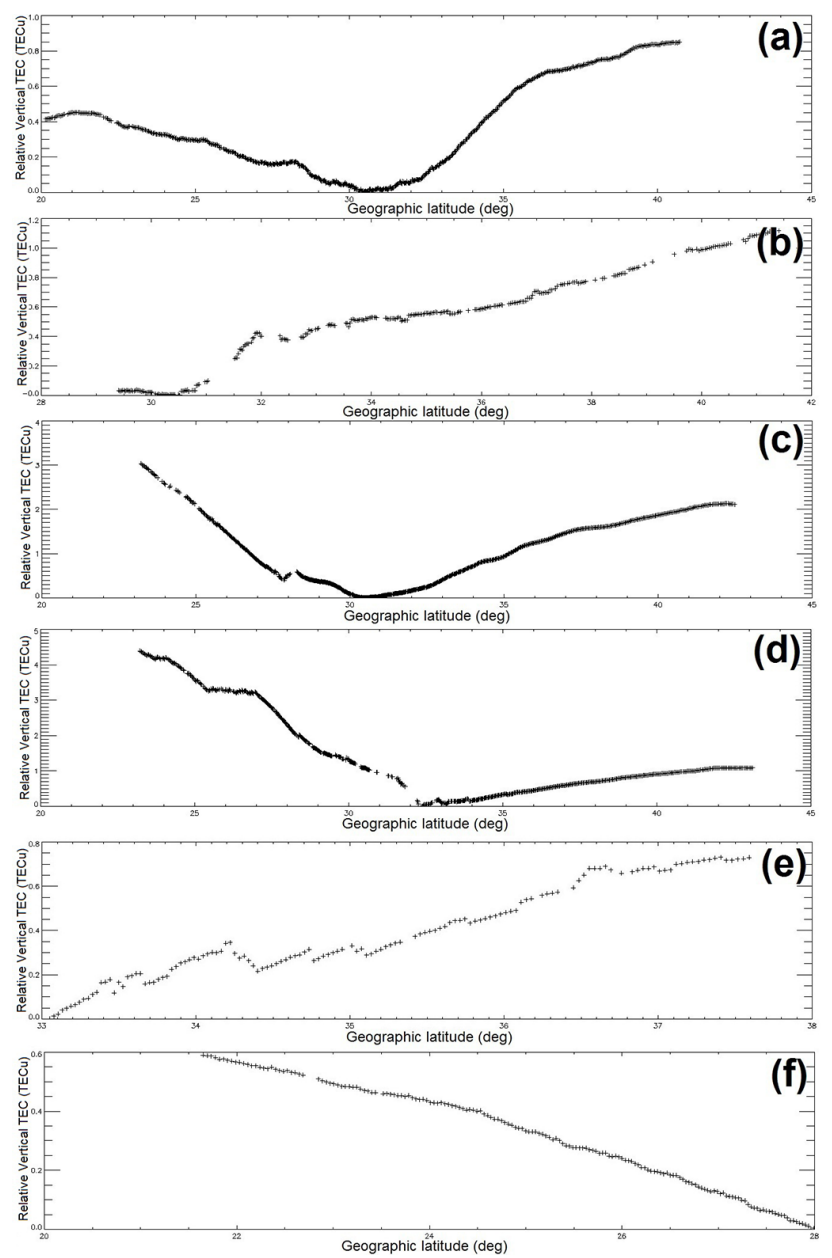

Figure 3. VTEC versus geographic latitude of the F region intercept. The passage time corresponds to the same panels (alphabetically sequenced) in Fig. 2.

07:32:41, 06:45:13, and 06:48 UT, respectively. These daytime emissions affected the lower ionosphere with hundreds of bursts in the band $1 \mathrm{kev}-6.2 \mathrm{MeV}$ (Tanaka et al., 2010; Mondal et al., 2012; Raulin et al., 2014). A high level of ionospheric perturbations were detected by CIDR over northern Africa and the eastern Mediterranean $\sim 6 \mathrm{~h}$ later between 23 and $28^{\circ}$, with $\Delta \mathrm{TEC} / \Delta t \leq 0.10 \mathrm{TECU} \mathrm{s}^{-1}$ at $12: 48 \mathrm{UT}$ (see Fig. 4c).

The visual examination of this passage found a structure in the $\Delta \mathrm{TEC} / \Delta t$ profile similar to the wave-like structure that occurred between 29 and $42^{\circ}$. It is worthwhile to mention that this is a signature of gravity waves created over the Anatolian plateau. This scenario is suggested by Garner et al. (2011), who verified that these kinds of ionospheric perturbations are associated with the orographic lift of the atmosphere over the Anatolian region, possibly through the propagation of the upward gravity waves. The amplitude of these waves is $\geq 0.01 \mathrm{TECU} \mathrm{s}^{-1}$, indicating that they would 

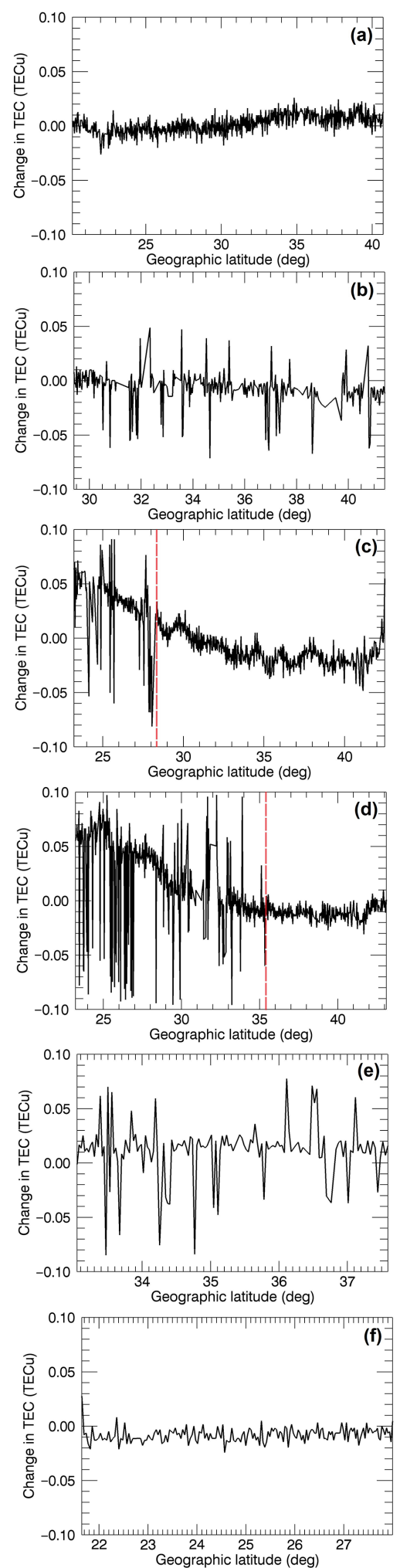

Figure 4. $\Delta \mathrm{TEC} / \Delta t$ versus geographic latitude of the $\mathrm{F}$ region intercept. The passage time corresponds to the same panels (alphabetically sequenced) in Fig. 2. The vertical red dashed lines show the disturbance upper limits during the main phase of the magnetar's flare. not generally be visible during more active ionospheric conditions.

The ionospheric perturbation continued, and even expanded, to much higher latitudes up to $35^{\circ}$ (see Fig. 4d). These structures resembled the poleward TIDs observed between 23 and $36^{\circ}$ at $14: 36$ UT. The upper latitude limit of the ionospheric disturbance detected during the main phase of the magnetar flare is indicated by the red dashed vertical lines in Fig. $4 \mathrm{c}$ and d. The comparison between the two upper limits defines the growth of the disturbed area, which expanded from 28 to $35^{\circ}$ within $1.8 \mathrm{~h}$. The TID's estimated virtual velocity is $385.8 \mathrm{~m} \mathrm{~s}^{-1}$, with $\Delta \mathrm{TEC} / \Delta t \leq 0.10 \mathrm{TECU} \mathrm{s}^{-1}$. The descending phase of the ionospheric perturbation observed between 33 and $37.5^{\circ}$ continued until 15:44 UT (Fig. 4e), then totally recovered on 23 January 2009 at 04:10 UT (Fig. 4f).

\section{Conclusions}

This paper presents convincing evidence that the source SGR J1550-5418 repeatedly caused significant ionospheric disturbances during the burst onset phase of the magnetar flare. The space weather data show that 22 January 2009 was a geomagnetically quiet day, with no effects of solar flares and geomagnetic storms on the ionosphere. The repeated bombardment of the high-energy radiations by that source was enough to enhance the electron number density of the ionosphere as computed by Mondal et al. (2012). The spatially relative TEC changes observed by the CIDR system reveal many interesting features of the magnetar's signature in the ionosphere. The northern equatorial fountain peak and gravity waves created over the Anatolian plateau are two ordinary phenomena resolved from VTEC latitudinal distribution. The onset phase of the magnetar flare was during the CIDR's nighttime satellite passage.

The nighttime small-scale perturbations detected by CIDR, with $\Delta \mathrm{TEC} / \Delta t \geq 0.05 \mathrm{TECU} \mathrm{s}^{-1}$, over the eastern Mediterranean on 22 January 2009 were synchronized with the onset phase of the magnetar flare and were consistent with the emission of hundreds of bursts from the source, as detected by BAT and INTEGRAL. It is well known that only the stronger bursts can be detected during daytime ionospheric conditions. In the present case, the emitted bursts would have produced an ionospheric disturbance large enough to be detected by CIDR during the daytime. The maximum large-scale perturbation was detected by CIDR, with $\Delta \mathrm{TEC} / \Delta t \leq 0.10 \mathrm{TECU} \mathrm{s}^{-1}$, over northern Africa and the eastern Mediterranean during the daytime $\sim 6 \mathrm{~h}$ after the main phase of the magnetar flare. These ionospheric perturbations resembled an unusual poleward TID caused by an extraterrestrial source. The TID's estimated virtual velocity was $385.8 \mathrm{~m} \mathrm{~s}^{-1}$, with $\Delta \mathrm{TEC} / \Delta t \leq 0.10 \mathrm{TECU} \mathrm{s}^{-1}$. The direction of the TID towards the North Pole suggests the opposite location of the ionizing source, which is in harmony 
with the sub-flare point (see Fig. 1). The proposed creation mechanism is similar to joule or particle heating, except that the ionizing radiation is X-ray emitted by the source SGR 1550-5418.

In general, CIDR observations appear as an interesting diagnostic tool of high-energy astrophysical bursts emitted by extraterrestrial sources. For magnetars, their ionospheric response complements their detection in space, in particular when space observations are not available due to detector saturation or Earth occultation.

Data availability. Short-time data are available. Kindly contact the author.

Competing interests. The author declares that he has no conflict of interest.

Acknowledgements. The ITNE CIDRs were constructed under the Defense University Research Instrumentation Program grant FA9550-04-1. The deployment of ITNE was supported by a USEgypt Joint Science and Technology Fund and the National Oceanic and Atmospheric Administration contract RA133D-07-SE-3966. The author also wishes to express his thanks to the anonymous reviewers and Mark Moldwin for their useful comments and review of the paper.

The topical editor, E. Yizengaw, thanks the three anonymous referees for help in evaluating this paper.

\section{References}

Bellm, E., Smith, D. M., and Hurley, K.: AXP 1E 1547.0-5408: RHESSI observations up to $400 \mathrm{keV}$, GCN Circ., http://gcn.gsfc. nasa.gov/gen/gen3/8857.gcn3 (last access: March 2017), 2009.

Bracewell, R. N. and Straker, T. W.: The study of solar flares by means of very long radio waves, Mon. Not. R. Astron. Soc., 109, 28-45, 1949.

Camilo, F., Ransom, S. M., Halpern, J. P., and Reynolds, J.: 1E 1547.0-5408: A Radio-emitting Magnetar with a Rotation Period of 2 Seconds, Astrophys. J., 666, L93, doi:10.1086/521826, 2007.

Connaughton, V. and Briggs, M.: AXP 1E1547.0-5408: Fermi GBM observations, GRB Coordinates Network, http://gcn.gsfc. nasa.gov/other/A.gen3 (last access: March 2017), 2009.

Dib, R., Kaspi, V. M., Scholz, P., and Gavriil, F. P.: RXTE Observation of Anomalous X-Ray Pulsar 1E 1547.0-5408 During and its 2008 and 2009 Outbursts, Astrophys. J., 748, 3, doi:10.1088/0004-637X/748/1/3, 2012.

Duncan, R. C. and Thompson, C.: Formation of very strongly magnetized neutron stars - Implications for gamma-ray bursts, Astrophys. J., 392, L9, doi:10.1086/186413, 1992.

Fishman, G. J. and Inan, U. S.: Observation of an ionospheric disturbance caused by a gamma-ray burst, Nature, 331, 418-420, doi:10.1038/331418a0, 1988.
Garner, T. W., Gaussiran, T. L., Tolman, B. W., Harris, R. B., Calfas, R. S., and Gallagher, H.: Total electron content measurements in ionospheric physics, Adv. Space Res., 42, 720-726, doi:10.1016/j.asr.2008.02.025, 2008.

Garner, T. W., Gaussiran, T. L., York, J. A., Munton, D. M., Slack, C. M., and Mahrous, A. M.: Ionospheric Tomography Network of Egypt: A New Receiver Network in Support of the International Heliophysical Year, Earth Moon Planets, 104, 227-235, doi:10.1007/s11038-008-9286-3, 2009.

Garner, T. W., Slack, C. M., Mehta, K., Scholze, A., and Mahrous, A. M.: Ionospheric structures correlated with Anatolian surface features, Radio Sci., 46, RS0D16, doi:10.1029/2011RS004653, 2011.

Gelfand, J. D. and Gaensler, B. M.: The Compact X-Ray Source 1E 1547.0-5408 and the Radio Shell G327.24-0.13: A New Proposed Association between a Candidate Magnetar and a Candidate Supernova Remnant, Astrophys. J., 667, 1111, doi:10.1086/520526, 2007.

Golenetskii, S., Aptekar, R., Mazets, E., Pal'shin, V., Frederiks, D., Oleynik, P., Ulanov, M., Svinkin, D., and Cline, T.: KonusWind detection of very bright SGR-like burst on January 25, 2009, GCN Circ., http://gcn.gsfc.nasa.gov/gcn/gcn3/8851.gcn3 (last access: March 2017), 2009.

Gronwall, C., Holland, S. T., Markwardt, C. B., Palmer, D. M., Stamatikos, M., and Vetere, L.: AXP 1E1547.0-5408 activity with SGR bursts, GCN Circ., http://gcn.gsfc.nasa.gov/gcn/gen3/8833. gcn3 (last access: April 2017), 2009.

Hernández-Pajares, M., Juan, J. M., and Sanz, J.: Medium-scale traveling ionospheric disturbances affecting GPS measurements: Spatial and temporal analysis, J. Geophys. Res., 111, A07S11, doi:10.1029/2005JA011474, 2006.

Inan, U. S., Lehtinen, N. G., Lev-Tov, S. J., Johnson, M. P., Bell, T. F., and Hurley, K.: Ionization of the lower ionosphere by gamma-rays from a Magnetar: Detection of a low energy (3-10 keV) component, Geophys. Res. Lett., 26, 3357, doi:10.1029/1999GL010690, 1999.

Inan, U. S., Lehtinen, N. G., Moore, R. C., Hurley, K., Boggs, S., Smith, D. M., and Fishman, G. J.: Massive disturbance of the daytime lower ionosphere by the giant gamma-ray flare from magnetar SGR 1806-20, Geophys. Res. Lett., 34, 8103, doi:10.1029/2006GL029145, 2007.

Israel, G. L., Esposito, P., Rea, N., Dall'Osso, S., Senziani, F., Romano, P., Mangano, V., Götz, D., Zane, S., Tiengo, A., Palmer, D. M., Krimm, H., Gehrels, N., Mereghetti, S., Stella, L., Turolla, R., Campana, S., Perna, R., Angelini, L., and De Luca, A.: The 2008 October Swift detection of X-ray bursts/outburst from the transient SGR-like AXP 1E 1547.0-5408, Mon. Not. R. Aastron. Soc., 408, 1387-1395, doi:10.1111/j.1365-2966.2010.17001.x, 2010.

Kouveliotou, C., Dieters, S., Strohmayer, T., van Paradijs, J., Fishman, G. J., Meegan, C. A., Hurley, K., Kommers, J., Smith, I., Frail, D., and Murakami, T.: An X-ray pulsar with a superstrong magnetic field in the soft big gamma-ray repeater SGR1806-20, Nature, 393, 235-237, doi:10.1038/30410, 1998.

Lamb, R. C. and Markert, T. H.: Gamma Ray Astronomy, Nebulae, Supernova Remnants, X Ray Sources, Neutron Stars, Point Sources, Proportional Counters, X Ray Telescopes, Astrophys. J., 244, 94-101, doi:10.1086/158688, 1981. 
Lin, L., Gogus, E., Baring, M. G., Granot, J., Kouveliotou, C., Kaneko, Y., Horst, A., Gruber, D., Kienlin, A., Younes, G., Watts, A. L., and Gehrels, N.: Broadband Spectral Investigation of SGR J1550-5418 Bursts, Astrophys. J., 756, 54, doi:10.1088/0004637X/756/1/54, 2012.

Mahrous, A. and Inoue, N.: Development of electromagnetic cascading in the Sun's magnetic field, Astron. Astrophys., 386, 308312, doi:10.1051/0004-6361:20020213, 2002.

Mahrous, A., Shimeis, A., and Garner, T.: First results of Coherent Ionospheric Doppler Receiver measurements over Egypt, Space Weather, 8, S07002, doi:10.1029/2009SW000548, 2010.

Mereghetti, S.: The strongest cosmic magnets: soft gamma-ray repeaters and anomalous X-ray pulsars, Astron. Astrophys. Rev., 15, 225-287, 2008.

Mereghetti, S., Gotz, D., Weidenspointner, G., von Kienlin, A., Esposito, P., Tiengo, A., Vianello, G., Israel, G. L., Stella, L., Turolla, R., Rea, N., and Zane, S.: Strong Bursts from the Anomalous X-Ray Pulsar 1E 1547.0-5408 Observed with INTEGRAL/SPI Anti-Coincidence Shield, Astrophys. J., 696, 74-78, doi:10.1088/0004-637X/696/1/L74, 2009.

Mondal, K., Chakrabarti, K., and Sasmal, S.: Detection of ionospheric perturbation due to a soft gamma ray repeater SGR J1550-5418 by very low frequency radio waves, Astrophys. Space Sci., 341, 259-264, doi:10.1007/s10509-012-1131-5, 2012.

Oyama, S. and Watkins, B.: Generation of atmospheric gravity waves in the polar thermosphere in response to auroral activity, Space Sci. Rev., 168, 463-473, doi:10.1007/s11214-011-9847-z, 2012.

Pacini, A. A. and Raulin, J.-P.: Solar X-ray flares and ionospheric sudden phase anomalies relationship: A solar cycle phase dependence, J. Geophys. Res., 111, 9301, doi:10.1029/2006JA011613, 2006.

Raulin, J.-P., Pacini, A. A., Kaufmann, P., Correia, E., and Martinez, M. A. G.: On the detectability of solar X-ray flares using Very Low Frequency sudden phase anomalies, J. Atmos. Sol.Terr. Phy., 68, 1029-1035, 2006.

Raulin, J.-P., David, P., Hadano, R., Saraiva, A. C. V., Correia, E., and Kaufmann, P.: The South America VLF NETwork (SAVNET), Earth Moon Planets, 104, 247-261, 2009.

Raulin, J.-P., Bertoni, F. C. P., Gaviln, H. R., Guevara-Day, W., Rodriguez, R., Fernandez, G., Correia, E., Kaufmann, P., Pacini, A. A., Stekel, T. R. C., Lima, W. L. C., Schuch, N. J., Fagundes, P. R., and Hadano, R.: Solar flare detection sensitivity using the South America VLF Network (SAVNET), J. Geophys. Res., 115, A07301, doi:10.1029/2009JA015154, 2010.

Raulin, J.-P., Trottet, G., de'Castro, C. G., Correia, E., and Macotela, E. L.: Nighttime sensitivity of ionospheric VLF measurements to X-ray bursts from a remote cosmic source, J. Geophys. Res., 119, 4758-4766, doi:10.1002/2013JA019670, 2014.

Scholz, P. and Kaspi, V. M.: The 2009 Outburst of Magnetar 1E 1547-5408: Persistent Radiative and Burst Properties, Astrophys. J., 739, 94, doi:10.1088/0004-637X/739/2/94, 2011.

Tanaka, Y. T., Raulin, J.-P., Bertoni, F. C., Fagundes, P. R., Chau, J., Schuch, N. J., Hayakawa, M., Hobara, Y., Terasawa, T., and Takahashi, T.: First Very Low Frequency Detection of Short Repeated Bursts from Magnetar SGR J1550-5418, Astrophys. J., 721, 24-27, 2010.
Terada, Y., Tashiro, M., Urata, Y., Endo, A., Onda, K., Kodaka, N., Morigami, K., Sugasahara, T., Iwakiri, W., Ohno, M., Kokubun, M., Suzuki, M., Takahashi, T., Yamaoka, K., Sugita, S., Enoto, T., Nakazawa, K., Makishima, K., Nakagawa, Y. E., Tamagawa, T., Uehara, T., Fukazawa, Y., Kira, C., Hanabata, Y., Sonoda, E., Yamauchi, M., Tanaka, H., Hara, R., Ohmori, N., Kono, K., Hayashi, H., Hong, S., and Vasquez, N.: AXP 1E1547.0-5408: suzaku WAM detections of the current activity, GRB Coordinates Network, 8845, 1, 2009.

Thompson, C. and Duncan, R. C.: The soft gamma repeaters as very strongly magnetized neutron stars - I. Radiative mechanism for outbursts, Mon. Not. R. Astron. Soc., 275, 255-300, 1995.

Thompson, C. and Duncan, R. C.: The Soft Gamma Repeaters as Very Strongly Magnetized Neutron Stars. II. Quiescent Neutrino, X-Ray, and Alfvén Wave Emission, Astrophys. J., 473, 322, doi:10.1086/178147, 1996.

Thompson, C., Lyutikov, M., and Kulkarni, S. R.: Electrodynamics of Magnetars: Implications for the Persistent X-Ray Emission and Spin-down of the Soft Gamma Repeaters and Anomalous XRay Pulsars, Astrophys. J., 574, 332, doi:10.1086/340586, 2002.

Thomson, N. R., Rodger, C. J., and Clilverd, M. A.: Large solar flares and their ionospheric D-region enhancements, J. Geophys. Res., 110, 6306-6316, 2005.

van der Horst, A. J., Kouveliotou, C., Gorgone, N. M., Kaneko, Y., Baring, M. G., Guiriec, S., Gogus, E., Granot, J., Watts, A. L., Lin, L., Bhat, P. N., Bissaldi, E., Chaplin, V. L., Finger, M. H., Gehrels, N., Gibby, M. H., Giles, M. M., Goldstein, A., Gruber, D., Harding, A. K., Kaper, L., von Kienlin, A., van der Klis, M., McBreen, S., Mcenery, J., Meegan, C. A., Paciesas, W. S., Peer, A., Preece, R. D., Ramirez-Ruiz, E., Rau, A., Wachter, S., Wilson-Hodge, C., Woods, P. M., and Wijers, R. A. M. J.: SGR J1550-5418 Bursts Detected with the FERMI Gamma-Ray Burst Monitor During its most Prolific Activity, Astrophys. J., 749, 122, doi:10.1088/0004-637X/749/2/122, 2012.

von Kienlin, A. and Connaughton, V.: AXP 1E1547.0-5408: Fermi GBM detection of additional bursts, GCN Circ., http://gcn.gsfc. nasa.gov/gcn/gcn3/8838.gcn3 (last access: March 2017), 2009.

von Kienlin, A., Gruber, D., Kouveliotou, C., Granot, J., Baring, M. G., Gogus, E., Huppenkothen, D., Kaneko, Y., Lin, L., Watts, A. L., Bhat, N. P., Guiriec, S., Horst, A. J., Bissaldi, E., Greiner, J., Meegan, C. A., Paciesas, W. S., Preece, R. D., and Rau, A.: Detection of Spectral Evolution in the Bursts Emitted During the 2008-2009 Active Episode of SGR J1550-5418, Astrophys. J., 755, 11, doi:10.1088/0004-637X/755/2/150, 2012.

Woods, P. M. and Thompson, C.: Compact Stellar X-ray Sources, edited by: Lewin, W. H. G. and van der Klis, M., Cambridge Univ. Press, Cambridge, 2006.

Younes, G., Kouveliotou, C., van der Horst, A. J., Baring, M. G., Granot, J., Watts, A. L., Bhat, P. N., Collazzi, A., Gehrels, N., Gorgone, N., Gogus, E., Gruber, D., Grunblatt, S., Huppenkothen, D., Kaneko, Y., von Kienlin, A., van der Klis, M., Lin, L., Mcenery, J., van Putten, T., and Wijers, R. A. M. J.: Time Resolved Spectroscopy of SGR J1550-5418 Bursts Detected with FERMI/Gamma Ray Burst Monitor, Astrophys. J., 785, 52, doi:10.1088/0004-637X/785/1/52, 2014. 